\title{
Extreme point properties of fixed-point sets
}

\section{Rodney Nillsen}

\begin{abstract}
We consider a semigroup $S$ acting as affine continuous maps on a compact convex set $X . F$ denotes the corresponding set of fixed points. Let $\operatorname{ex} X$ and exF denote the corresponding sets of extreme points. If $X$ is a simplex, conditions are given which ensure that when $x \in F$, the maximal measure representing $x$ is invariant under $S$. We also prove exF $=F \cap$ exX under conditions involving extreme amenability of $S$. Topological properties of exF are also studied.
\end{abstract}

1. Definitions and notation

$S$ will denote a semigroup and $L_{\infty}$ the space of all bounded real valued functions on $S$. For $s \in S$ and $f \in L_{\infty}, I_{s} f \in L_{\infty}$ is defined by: $\quad z_{s} f(t)=f(s t)$. $L$ denotes a closed subspace of $L_{\infty}$, containing the constant functions and such that $l_{s} L \subseteq L$ for each $s \in S$. By a mean $\mu$ on $L$ is meant an element $\mu \in L^{*}$ such that $\mu \geq 0$ and $\mu(1)=1$ (hence $\|\mu\|=1)$. Such a mean $\mu \in L^{*}$ is called left invariant if $l_{s}^{*} \mu=\mu$ for all $s \in S$. When such a $\mu$ exists, $L$ is said to be left amenable. If $L$ is an algebra and a left invariant $\mu$ exists which is also multiplicative (that is, $\mu(f g)=\mu(f) \mu(g)$ for $f, g \in L$ ), $L$ is said to be extremely left amenable. $S$ is said to be (extremely) left amenable if

Received 25 October 1971. These results form part of the author's Ph.D. thesis at The Flinders University of South Australia, prepared under the supervision of Professor 1. Kluvanek; see also Bull. Austral. Math. Soc. 6 (1972), 315-316. The author was supported by a Commonwealth Postgraduate Award. 
$L_{\infty}$ is (extremely) left amenable. $M$ denotes the set of means on $L$ - it is a compact convex set in the weak* topology .

$X$ shall denote a compact convex set situated in some Hausdorff locally convex topological vector space $E$. $A(X)$ denotes all affine continuous real valued functions on $X . K(X)$ denotes all convex continuous real valued functions on $X$. A representation of $S$ as a semigroup of continuous affine maps on $X$ is assumed to be given. We define $F$ to be $\{x \in X: s x=x$ for all $s \in S\}$, and it is the fixed point set associated with the given representation of $S . F$ is a compact convex subset of $X$. exX and exF denote the sets of extreme points of $X$ and $F$ respectively.

Given $z \in X$, define $T_{z}: A(X) \rightarrow L_{\infty}$ by $\left(T_{z} f\right)(s)=f(s z)$ for $f \in A(X), s \in S \cdot T_{z}$ is a positive linear operator of norm 1 . If $z \in X$ is such that $T_{z}(A(X)) \subseteq L, T_{z}^{*}$ maps $L^{*}$ into $A(X) *$ - in fact if $\mu$ is a mean on $L, T_{z}^{*} \mu$ will be a mean on $A(X)$ and corresponds to an evaluation given by a point of $X$ ([1]). Hence if $T_{z}(A(X)) \subseteq L, T_{z}^{*}$ can be regarded as mapping the means on $L$ into $X$.

For information on Choquet simplices etc., see [16]. For K-analytic and $K$-Borel sets see [13].

\section{The extremely amenable case}

LEMMA 2.1 ([1]). Let $\mu$ be a left invariant mean on $L$ and $z \in X$ be such that $T_{z}(A(X)) \subseteq L$. Then $T_{z}^{*} \mu E$. If $S$ is left anenable, $F$ is non void.

Proof. For each $f \in A(X)$ and each $s \in S$ we have:

$$
f\left(s \cdot T_{z}^{*} \mu\right)=\mu\left(T_{z}(f \circ s)\right)=\mu\left(\tau_{s}\left(T_{z} f\right)\right)=\mu\left(T_{z} f\right)=f\left(T_{z}^{*} \mu\right) .
$$

Since $A(X)$ separates points of $X, s\left(T_{z}^{*} \mu\right)=T_{z}^{*} \mu$ for all $s \in S$; that is, $T_{z}^{*} \mu \in F$.

The last statement is due to Day [5], and follows from $T_{z}(A(X)) \subseteq L_{\infty}$ for all $z$. 
For a given compact Hausdorff space $T, p(T)$ shall denote all regular Borel probability measures on $T$. We define

$$
I=\left\{\mu \in p(X): \mu \circ s^{-1}=\mu \text {, all } s \in S\right\} .
$$

$I$ is a weak* compact convex subset of $p(X)$, and $F$ is non void if and only if $I$ is non void. Given $v \in X$ let $S_{v}=\{s \in S: s v \in \operatorname{ex} X\}$.

Define $S_{0}=\prod_{v \in \operatorname{exX}} S_{v} \cdot S_{0}$ is a subsemigroup of $S \cdot$ A subset $S_{1}$ of $S$ is said to be left near thick if, given $t \in S$, there is $s \in S_{1}$ such that $t s \in S_{1}$.

LEMMA 2.2. Let $X$ be a Choquet simplex and suppose that $S_{0}$ is left near thick in $S$. Let $x \in X$ and $\mu \in p(X)$ be its (unique) representing maximal measure. Then if $x \in F, \mu \in I$; and if $x \in \operatorname{ex} F$, $\mu \in \operatorname{exI}$.

Proof. By Theorem I of Jellett [12], if $s(\operatorname{ex} X) \subseteq \operatorname{exX}$ and $\mu \in p(X)$ is maximal, then $\mu \circ s^{-1}$ is maximal. Hence if $x \in F$ and $\mu$ is its representing maximal measure, $\mu \circ s^{-1}$ is maximal and represents $s x=x$, provided $s \in S_{0}$. By the uniqueness of representing maximal measures, $\mu=\mu \circ s^{-1}$ for all $s \in S_{0}$.

Now if $t \in S$ choose $s \in S_{0}$ such that $t s \in S_{0}$. Then

$$
\mu \circ t^{-1}=\mu \circ s^{-1} \circ t^{-1}=\mu \circ(t s)^{-1}=\mu
$$

as $t s \in S_{0} ;$ that is, $\mu \in I$.

If now $x \in \operatorname{exF}$, let $\mu$ be its representing maximal measure. Let $\mu_{1}, \mu_{2} \in I$ and $0<\lambda<1$ be such that $\mu=\lambda \mu_{1}+(1-\lambda) \mu_{2}$. If $f \in K(X), \mu$ maximal implies $\mu(\bar{f}-f)=0$. Since $\bar{f}-f \geq 0$, we have $\mu_{1}(\bar{f}-f)=\mu_{2}(\bar{f}-f)=0$. Hence for each $f \in K(X), \mu_{1}(\bar{f})=\mu_{1}(f)$ and $\mu_{2}(\bar{f})=\mu_{2}(f)$. It now follows (Phelps [17], p. 64) that $\mu_{1}$ and $\mu_{2}$ are maximal. Let $x_{1}, x_{2}$ respectively be the resultants of $\mu_{1}, \mu_{2}$. Because $\mu_{1}, \mu_{2} \in I, x_{1}, x_{2} \in F$, and $x=\lambda x_{1}+(1-\lambda) x_{2}$. Since $x \in \operatorname{ex} F$, 
$x=x_{1}=x_{2}$. By uniqueness of representing maximal measures, $\mu=\mu_{1}=\mu_{2}$. Hence $\mu \in \operatorname{ex} T$.

LEMMA 2.3. Let $L$ be left amenable. Let $\mu \in I$ be such that, for each $f, g \in C(X)$, the function on $S$ defined by $s \rightarrow \mu((f \circ s) g)$ belongs to $L$. Then the following are equivalent:

(1) $\mu \in \operatorname{exI}$;

(2) for each left invariant mean $m$ on $L$ and $f, g \in C(X)$, $m(s \rightarrow \mu((f \circ s) g))=\mu(f) \mu(g) ;$

(3) for some mean $m$ on $L$ we have $m(s \rightarrow \mu((f \circ s) g))=\mu(f) \mu(g)$ for azz $f, g \in C(X)$.

Proof. This can be adapted from [4], Theorem 2.1.

THEOREM 2.4. Suppose that:

(1) $L$ is left comenable;

(2) each element of $S$ has a right zero;

(3) $X$ is a Choquet simplex;

(4) $S_{0}$ is left near thick in $S$;

(5) for $\mu \in I$ and $f, g \in C(X)$, the function $s \rightarrow \mu((f \circ s) g)$ bezongs to $L$.

Then $F \cap \operatorname{exX}=\operatorname{ex} F$.

Proof. We obviously have $\operatorname{ex} X \cap F \subseteq \operatorname{ex} F$. Conversely let $x \in \operatorname{ex} F$ and let $\mu \in p(X)$ be its representing maximal measure. By Lemma 2.2, $\mu \epsilon \operatorname{exI}$ and Lemma 2.3 gives that for each left invariant mean $m$ on $L$, and $f, g \in C(X), m(s+\mu((f \circ s) g))=\mu(f) \mu(g)$. By (2) choose $t \in S$ such that $s t=t$. Then $\mu((f \circ s) g)=\mu((f g) \circ t)=\mu(f g)$. Then for $f, g \in C(X), \mu(f g)=\mu(f) \mu(g)$. Hence there is $x_{0} \in X$ such that $f\left(x_{0}\right)=\mu(f)$ for $f \in C(X)$; that is, $\mu=\varepsilon_{x_{0}}$. Because $\mu$ was chosen to be maximal, $x_{0} \in \operatorname{exX}$ (Phelps [17], p. 8); and for $f \in A(X)$,

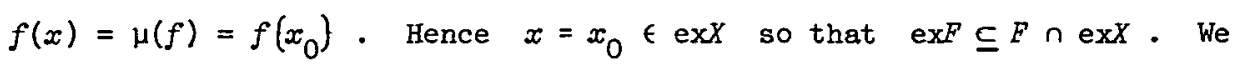


deduce $\operatorname{ex} F=F \cap \operatorname{exl}$.

COROLLARY 2.5. A semigroup $S$ is extremely left amenable if and only if $S$ is left amenable and each element of $S$ has a right zero.

Proof. If $S$ is extremely left amenable, Granirer [8] has shown that any finite subset of $S$ has a common right zero. Hence each element of $S$ has a right zero. Conversely, apply Theorem 2.4. For $X$ take the means on $L_{\infty}$. Represent $S$ as a semigroup of continuous affine maps on $X$ by: $s \rightarrow Z_{s}^{*}, s \in S \cdot \operatorname{exX}$ is the set of multiplicative means on $L_{\infty}$ and $l_{s}^{*}(\operatorname{exX}) \subseteq \operatorname{exX}$ for all $s \in S$ is clear. All the conditions of Theorem 2.4 hold so we may deduce that $\mu \in \dot{e x} X$ exists such that $z_{s}^{*} \mu=\mu$ for all $s \in S$. But this simply says that $S$ is extremely left amenable.

REMARKS. (1) In view of the characterizations in [8] and [13], p. 66 , the result of the corollary is that a semigroup has the common fixed point property on compacta if and only if it has the individual fixed point property on any set and the common fixed point property (continuous affine maps) on compact convex sets. There are many semigroups in which each element has a right zero but which are not left amenable. For example, for each natural number $n$, let $S_{n}$ be the semigroup of continuous maps from the closed unit sphere of Euclidean $n$-space into itself. The Brouwer fixed point theorem ensures that each element of $S_{n}$ has a right zero. However, $s_{n}$ is obviously not extremely left amenable. It follows that $s_{n}$ is not left amenable, $n \stackrel{0}{=} 1,2,3, \ldots$.

(2) The conditions of Theorem 2.4 are fulfilled if $S$ is extremely left amenable, $X$ is a simplex and $s(\operatorname{ex} X) \subseteq \operatorname{ex} X$. The conclusion $\operatorname{ex} F=F \cap \operatorname{exX}$ then extends the main result of Mitchell [15] and Theorem 6 (b) of Granirer [9].

\section{Topological properties of exp}

The action of $S$ on $X$ is said to be weakly almost periodic if for each $f \in C(X),\{f \circ s: s \in S\}$ is relatively weakly compact in $C(X)$. We introduce $M_{0}$, the set of maximal measures in $p(X)$. Define $I_{0}=I \cap M_{0} \cdot r: p(X) \rightarrow X$ denotes the resultant map, and it is 
continuous. We give conditions which ensure that $F$ is a Choquet simplex. We are also interested in the way in which the properties of exX influence those of exF.

THEOREM 3.1. Suppose that $X$ is a Choquet simplex and that $S_{0}$ is left near thick in $S$. Then $F$ is a Choquet simplex.

Proof. The resultant map $r: I_{0} \rightarrow F$ is injective and by Lemma 2.2 it is also onto $F \cdot r$ is also affine so that $F$ will be a choquet simplex if $I_{0}$ is a Choquet simplex. Let $\lambda, \mu \in \tilde{I}_{0}=\left\{\alpha \gamma: \alpha \geq 0, \gamma \in I_{0}\right\}$. Then $\lambda, \mu \in \tilde{I}=\{\alpha \gamma: \alpha>0, \gamma \in I\}$ and $I$ is a simplex (Phelps [17], p. 80). Let $\gamma_{0}$ be the greatest lower bound in $\tilde{I}$ of $\lambda, \mu \in \tilde{I}$. Then it follows from $[17], p .65$, that $\gamma_{0}$ is inaximal (since $\gamma_{0} \leq \lambda$ in the usual ordering). So $\gamma_{0} \in I_{0}$ and we immediately see that $\gamma_{0}$ is the greatest lower bound of $\lambda, \mu$ in $I_{0}$. Hence $I_{0}$ is a simplex. By our earlier remarks, $F$ is a simplex.

LEMMA 3.2. Let $\operatorname{exX}$ be a $K$-Borel set in $X$. Then $M_{0}$ is a $K$-Borel set in $p(X)$. (Of course $p(X)$ has the weak* topology in which it is a compact Hausdorff space.)

Proof. We introduce the family $A$ whose elements are Borel subsets of $X$. We define a Borel set $A$ in $X$ to be in $A$ if and only if for each real $\alpha,\{\mu \in p(X): \mu(A) \geq \alpha\}$ is $K$-Borel in $p(X)$. If $A \subseteq X$ is compact then $\{\mu \in p(X): \mu(A) \geq \alpha\}$ is compact in $p(X)$ for all real $\alpha$. Hence $A$ contains the compact subsets of $X$.

Now let $\left(A_{i}\right)$ be a sequence in $A$ such that $A_{i} \downarrow A$. Then $\{\mu \in p(X): \mu(A) \geq \alpha\}$ is simply $\prod_{1}^{\infty}\left\{\mu \in p(X): \mu\left(A_{i}\right) \geq \alpha\right\}$, which is $K$-Borel as $A_{i} \in A$ for all $i$. If $\left(B_{i}\right)$ is a sequence in $A$ with $B_{i} \uparrow B$, for each $(m, n)$ define $K_{(m, n)}=\left\{\mu \in p(X): \mu\left(B_{m}\right) \geq \alpha-\frac{1}{n}\right\}$.

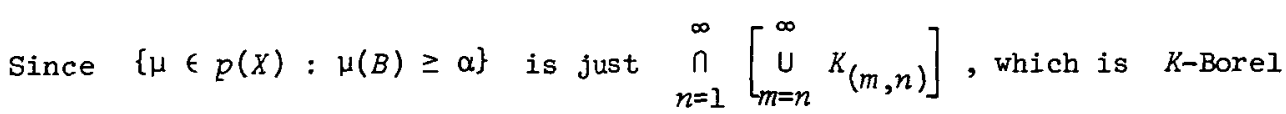
as $B_{i} \in A$, we must have $B \in A$. 
We have now shown that $A$ contains compact sets and is closed under countable increasing unions and countable decreasing intersections. Because the compact sets are closed under finite unions and finite intersections it follows (in much the same way that Halmos [10] proves his Theorem B, p. 27) that A contains the smallest family closed under countable unions and intersections and containing the compact sets; that is, $A$ contains the $K$-Borel sets. Hence for each $K$-Borel set $A$, $\{\mu \in p(X): \mu(A)=1\}$ is $K$-Borel in $p(X)$. Hence $\{\mu \in p(X): \mu(\operatorname{ex} X)=1\}$ is $K$-Borel in $p(X)$. However this set is just $M_{\mathrm{O}} \quad([17]$, p. 122$)$.

\section{THEOREM 3.3. Suppose that}

(1) $X$ is a Choquet simplex;

(2) L is left comenable;

(3) $s_{0}$ is left near thick in $s$;

(4) the action of $S$ on $X$ is weakly almost periodic;

(5) for $\mu \in I$ and $f, g \in C(X)$ the function $s \rightarrow \mu((f \circ s) g)$ belongs to $L$.

Then if exX is compact, exF is compact. If exX is K-Borel in $X$, exF is K-analytic in $F$.

Proof. We may clearly assume that $F$ is non void. We have

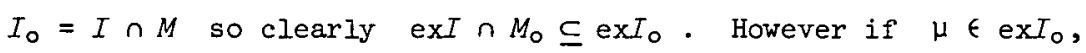
$\mu_{1}, \mu_{2} \in I$ and $0<\lambda<1$ with $\mu=\lambda \mu_{1}+(1-\lambda) \mu_{2}$, then $\mu_{1}, \mu_{2} \in M_{0}$ (since $\gamma \in p(X)$ is maximal if and only if $\gamma(\bar{f}-f)=0$ for all $f \in K(X),[17]$, p. 64). $\mu \in$ exIo now gives $\mu=\mu_{1}=\mu_{2}$. Hence $\operatorname{ex} I_{0} \subseteq \operatorname{ex} I \cap M_{0}$. We deduce that $\operatorname{ex} I_{0}=\operatorname{exI} \cap M_{0}$.

Now the proof of Theorem 4.5 of [4] can be modified so that when applied in this situation we deduce that exI is compact. If exX is compact, $M_{0}=\{\mu \in p(X): \mu(\operatorname{ex} X)=1\}$ is compact, so that ex $I_{0}$ is compact. Then exF, being the continuous image of exIo under the resultant map, is compact.

If exX is $K$-Borel, Lerma 3.2 gives that $M_{0}$ is $K$-Borel. Hence ex $I$ is $K$-Borel in $I$. exF is now $K$-analytic, because it is the 
continuous image of exIo under the resultant map (see [2]).

- REMARKS. The conditions of Theorem 3.3 are satisfied if $G$ is an equi-continuous group of affine homeomorphisms of the simplex. In this case the rôle of $L$ is played by the bounded almost periodic functions on $G$ - this admits a left invariant mean by [11], p. 250.

\section{References}

[1] L.N. Argabright, "Invariant means and fixed points; a sequel to Mitchell's paper", Trans. Amer. Math. Soc. 130 (1968), 127-130.

[2] D.W. Bressler and M. Sion, "The current theory of analytic sets", Canad. J. Math. 16 (1964), 207-230.

[3] Gustave Choquet, Lectures on analysis, Volumes I, II, III (W.A. Benjamin, New York, Amsterdam, 1969).

[4] George Converse, Isaac Namioka and R.R. Phelps, "Extreme invariant positive operators", Trans. Amer. Math. Soc. 137 (1969), 375-385.

[5] Mahlon M. Day, "Fixed-point theorems for compact convex sets", IZZinois J. Math. 5 (1961), 585-590.

[6] Nelson Dunford and Jacob T. Schwartz, Linear operators, Part I (Interscience [John Wiley \& Sons], New York, London, 1958).

[7] Edward G. Effros, "Structure in simplexes", Acta Math. 117 (1967), 103-121.

[8] E. Granirer, "Extremely amenable semigroups", Math. Scand. 17 (1965), 177-197.

[9] E. Granirer, "Extremely amenable semigroups II", Math. Scand. 20 (1967), 93-113.

[10] Paul R. Halmios, Measure theory (Van Nostrand, Toronto, New York, London, 1950). 
[11] Edwin Hewitt and Kenneth A. Ross, Abstract harmonic analysis, Vol. I (Die Grundlehren der mathematischen Wissenschaften, Band 115. Academic Press, New York; Springer-Verlag, Berlin, Göttingen, Heidelberg, 1963).

[12] Francis Jellett, "Homomorphisms and inverse limits of Choquet simplexes", Math. Z. 103 (1968), 219-226.

[13] E.S. Ljapin, Semigroups (Translations of Mathematical Monographs, 3. Amer. Math. Soc., Providence, Rhode Island, 1963).

[14] Paul A. Meyer, Probability and potentials (Blaisdell, Waltham, Massachusetts; Toronto; London; 1966).

[15] Theodore Mitchell, "Fixed points and multiplicative left invariant means", Trans, Amer. Math. Soc. 122 (1966), 195-202.

[16] Theodore Mitchell, "Function algebras, means, and fixed points", Trans. Amer. Math. Soc. 130 (1968), 117-126.

[17] Robert R. Phelps, Lectures on Choquet's theorem (Van Nostrand, Princeton, New Jersey; Toronto; New York; London; 1966).

Flinders University of South Australia, Bedford Park, South Australia, and

The Royal University of Malta, Msida, Malta. 